\title{
The Polymeal: a more natural, safer, and probably tastier (than the Polypill) strategy to reduce cardiovascular disease by more than $75 \%$
}

\author{
Oscar H Franco, Luc Bonneux, Chris de Laet, Anna Peeters, Ewout W Steyerberg, \\ Johan P Mackenbach
}

\begin{abstract}
Objective Although the Polypill concept (proposed in 2003) is promising in terms of benefits for cardiovascular risk management, the potential costs and adverse effects are its main pitfalls. The objective of this study was to identify a tastier and safer alternative to the Polypill: the Polymeal.

Methods Data on the ingredients of the Polymeal were taken from the literature. The evidence based recipe included wine, fish, dark chocolate, fruits, vegetables, garlic, and almonds. Data from the Framingham heart study and the Framingham offspring study were used to build life tables to model the benefits of the Polymeal in the general population from age 50, assuming multiplicative correlations. Results Combining the ingredients of the Polymeal would reduce cardiovascular disease events by $76 \%$. For men, taking the Polymeal daily represented an increase in total life expectancy of 6.6 years, an increase in life expectancy free from cardiovascular disease of 9.0 years, and a decrease in life expectancy with cardiovascular disease of 2.4 years. The corresponding differences for women were 4.8, 8.1, and 3.3 years.
\end{abstract}

Conclusion The Polymeal promises to be an effective, non-pharmacological, safe, cheap, and tasty alternative to reduce cardiovascular morbidity and increase life expectancy in the general population.

\section{Introduction}

Cardiovascular disease continues to be the leading cause of mortality and morbidity in Western populations. ${ }^{1}$ Although several risk factors for cardiovascular disease have been identified, its prevention is still suboptimal owing to high costs, low compliance, and side effects of treatment. In 2003 Wald and Law introduced the concept of the Polypill. ${ }^{2}$ The advocates of the Polypill selected six pharmacological components that by modifying different risk factors of cardiovascular disease multiplicatively might reduce the levels of cardiovascular disease in the population by more than $80 \% .^{2}$ In general, the medical community has welcomed the concept but questioned the potential adverse effects and costs of such an intervention.

Our objective was to define a safer, nonpharmacological, and tastier alternative to the Polypill in the general population: the Polymeal. We also wanted to calculate the potential effects of the Polymeal in terms of total life expectancy and life expectancy with and without cardiovascular disease.

\section{Methods}

\section{The recipe}

To optimise the Polymeal ingredients we used an evidence based diet conceptual framework, which follows similar principles to evidence based medicine. ${ }^{4}$ The constituting elements of a meal or recipe are selected on the basis of the best available evidence; the evidence available for each ingredient is graded according to the level of evidence. We searched PubMed, informed by expert advice, for nonpharmacological ingredients with evidence levels 1 or 2: randomised controlled trials, meta-analyses of randomised controlled trials, and meta-analyses of observational studies. ${ }^{5}$ To be included in the Polymeal, the ingredient had to have individually reported effects (not as an element of a diet) on reduction in cardiovascular disease events or modification of risk factors for cardiovascular disease. We checked papers retrieved for further possible ingredients. The following dietary elements met the inclusion criteria to be ingredients of the Polymeal: wine, fish, dark chocolate, fruits and vegetables, almonds, and garlic (Allium sativum).

\section{Efficacy of the Polymeal}

We obtained information from the literature on the benefits of the interventions (table 1). Daily consumption of $150 \mathrm{ml}$ of wine reduces cardiovascular disease by $32 \%$ (95\% confidence interval $33 \%$ to $41 \%){ }^{6}$ Fish $(114 \mathrm{~g})$ consumed four times a week reduces cardiovascular disease by $14 \%$ ( $8 \%$ to $19 \%){ }^{7}$ For chocolate, fruits and vegetables, almonds, and garlic, we found data on modification of risk factors for cardiovascular disease. One hundred grams of dark chocolate consumed daily reduces systolic blood pressure by $5.1 \mathrm{~mm} \mathrm{Hg}$ and diastolic blood pressure by 1.8 $\mathrm{mm} \mathrm{Hg}^{8}$; similar reductions in blood pressure correspond to a reduction in cardiovascular disease events of $21 \%$ (14\% to $27 \%){ }^{9}$ A total of $400 \mathrm{~g}$ of fruit and vegetables consumed daily produced a reduction in blood pressure similar to that observed with chocolate $(4.0 \mathrm{~mm} \mathrm{Hg}$ systolic blood pressure and $1.5 \mathrm{~mm}$ $\mathrm{Hg}$ diastolic blood pressure), so we decided to assume

\begin{tabular}{|c|c|c|}
\hline Ingredients & $\begin{array}{l}\text { Percentage reduction } \\
(95 \% \mathrm{CI}) \text { in risk of CVD }\end{array}$ & Source \\
\hline$\overline{\text { Wine (150 ml/day) }}$ & 32 (23 to 41$)$ & Di Castelnuovo et al $(\mathrm{MA})^{6}$ \\
\hline Fish (114 g four times/week) & 14 (8 to 19$)$ & Whelton et al $(\mathrm{MA})^{7}$ \\
\hline Dark chocolate (100 g/day) & 21 (14 to 27$)$ & Taubert et al $(\mathrm{RCT})^{8}$ \\
\hline Fruit and vegetables (400 g/day) & 21 (14 to 27$)$ & John et al $(\mathrm{RCT})^{10}$ \\
\hline Garlic (2.7 g/day) & 25 (21 to 27$)$ & Ackermann et al $(\mathrm{MA})^{11}$ \\
\hline Almonds (68 g/day) & 12.5 (10.5 to 13.5$)$ & Jenkins et al (RCT), ${ }^{15}$ Sabate et al (RCT) ${ }^{16}$ \\
\hline Combined effect & 76 (63 to 84 ) & \\
\hline
\end{tabular}

$\mathrm{CVD}=$ cardiovascular disease; $\mathrm{MA}=$ meta-analysis; $\mathrm{RCT}=$ =randomised controlled trial.
Department of Public Health, Erasmus MC

University Medical Centre Rotterdam, PO Box 1738, 3000 DR Rotterdam,

Netherlands

Oscar H Franco Chris de Laet

Ewout W

Steyerberg

associate professor

Johan P

Mackenbach

professor

Belgian Health Care Knowledge Centre (KCE),

Wetstraat 155,

B-1040, Brussels,

Belgium

Luc Bonneux

senior researcher

Department of

Epidemiology and

Preventive

Medicine, Monash

University Central

and Eastern Clinica

School, Melbourne,

Australia

Anna Peeters

senior researcher

Correspondence to:

O H Franco

o.francoduran@

erasmusmc.nl

BMJ 2004;329:1147-50 scientific researcher senior researcher 
Table 2 Lifetime effect (years) of Polymeal at age 50, stratified by sex

\begin{tabular}{|c|c|c|c|c|c|c|}
\hline \multirow[b]{2}{*}{ Intervention } & \multicolumn{2}{|c|}{ Total life expectancy } & \multicolumn{2}{|c|}{$\begin{array}{l}\text { Life expectancy } \\
\text { free from CVD }\end{array}$} & \multicolumn{2}{|c|}{ Life expectancy with CVD } \\
\hline & Effect & Difference & Effect & Difference & Effect & Difference \\
\hline \multicolumn{7}{|l|}{ Men: } \\
\hline None (overall) & 28.7 & Ref & 21.0 & Ref & 7.7 & Ref \\
\hline Polymeal & 35.2 & 6.6 & 30.0 & 9.0 & 5.3 & -2.4 \\
\hline \multicolumn{7}{|l|}{ Women: } \\
\hline None (overall) & 34.2 & Ref & 26.9 & Ref & 7.3 & Ref \\
\hline Polymeal & 39.0 & 4.8 & 35.0 & 8.1 & 4.0 & -3.3 \\
\hline
\end{tabular}

$\mathrm{CVD}=$ cardiovascular disease; Ref=reference value.

the same reduction in cardiovascular disease effect as assigned for chocolate $(21 \%){ }^{10}$

Daily consumption of garlic reduced total cholesterol concentrations by $0.44 \mathrm{mmol} / \mathrm{l}(17.1 \mathrm{mg} / \mathrm{dl}),{ }^{11}$ corresponding to $66 \%$ of the reduction $(0.66 \mathrm{mmol} / \mathrm{l})$ that was found to be associated with a 38\% reduction in cardiovascular disease at age $50 .^{13}$ Therefore, we considered $66 \%$ of the effect previously reported and assumed a reduction of $25 \%(21.7 \%$ to $27.7 \%)$ in cardiovascular disease events for garlic. Most of the randomised controlled trials included in the metaanalysis used 600-900 mg/day of dried garlic powder preparations, equivalent to $1.8-2.7 \mathrm{~g}$ /day of fresh garlic. ${ }^{14}$ We selected $2.7 \mathrm{~g}$ /day of fresh garlic for the Polymeal. Consuming $68 \mathrm{~g}$ /day of almonds produced half the reduction in total cholesterol $(10 \mathrm{mg} / \mathrm{dl})$ observed with garlic, ${ }^{15}{ }^{16}$ so we assumed a reduction in cardiovascular disease half the one assigned to garlic.

We calculated the combined effect of the ingredients of the evidence based diet Polymeal by multiplying their correspondent relative risk estimates. This is the same method that was used for the Polypill. ${ }^{2}$

\section{Study population}

We applied the effects of the Polymeal to a life table built using the Framingham study population. The original Framingham heart study cohort consisted of 5209 respondents (2336 men) residing in Framingham, Massachusetts, between 1948 and 1951. Participants have been examined biannually, and the cohort has been followed for 46 years. ${ }^{17}$

We used follow up data from participants attending study examinations 4 (1956-8), 11 if present or otherwise 12 (1969-73), and 19 if present or otherwise 20 (1985-9). Follow up started at the date of the chosen baseline examination. Each participant could therefore be included more than once but for different follow up periods of no more than 12 years in order to avoid overlapping periods. A total of 9181 participantobservation periods of follow up were available for the analysis.

We used three endpoints in this study: the composite endpoint of incident non-fatal cardiovascular disease (angina, coronary insufficiency, myocardial infarction, congestive heart failure, stroke, transient ischaemic attack, and intermittent claudication), fatal cardiovascular disease, and other causes of death. In the Framingham heart study, a panel of three physicians evaluated all events (fatal and non-fatal); agreement of all three was needed. ${ }^{18}$ We selected total cardiovascular disease as the outcome (and not coronary heart disease and stroke separated) on the basis of current recommendations in the European guidelines on cardiovascular disease prevention. ${ }^{19}$
Effects of the Polymeal on life expectancy and time with cardiovascular disease

To translate the effects of the Polymeal on reduction of cardiovascular disease events (table 1) in terms of differences in life expectancy and life expectancy with and without cardiovascular disease, we created multi-state life tables starting at age 50 years and closing at 100 years of age. We stratified the multi-state life tables by sex and created them separately for the general population with and without the Polymeal. The multi-state life tables included three different states: "free from cardiovascular disease," "history of cardiovascular disease," and "death." The possible transitions were from "free from cardiovascular disease" to "history of cardiovascular disease" or "death" and from "history of cardiovascular disease" to "death." ${ }^{20}$ In the life tables representing the population with the Polymeal, we derived the effects by decreasing the rates for the transitions "free from cardiovascular disease" to "history of cardiovascular disease" and "history of cardiovascular disease" to "death" by the estimated risk reduction associated with the Polymeal. We used Excel spreadsheets for all analyses.

\section{Results}

\section{Effects of the Polymeal}

Combining all the ingredients of the Polymeal resulted in cardiovascular disease being reduced by $76 \%(95 \%$ confidence interval $63 \%$ to $84 \%$ ) (table 1). Whether increasing the amount of each ingredient would increase the effect of the Polymeal is uncertain. On the other hand, decreasing the quantities could be expected to reduce the effects of the Polymeal. Omitting wine from the Polymeal had the strongest effect on the risk reduction of cardiovascular disease (from $76 \%$ to $65 \%$ ). Excluding any of the other ingredients had a lesser effect: $73 \%$ reduction without fish, $70 \%$ without chocolate or fruits and vegetables, $68 \%$ without garlic, and $73 \%$ without almonds.

\section{Lifetime effects of the Polymeal}

The effect of the Polymeal represented a large increase in total life expectancy and life expectancy free from cardiovascular disease and a decrease in life expectancy with cardiovascular disease for both men and women (table 2). For men, taking the Polymeal would result in increases of 6.6 years in total life expectancy and 9.0 years in life expectancy free from cardiovascular disease. The decrease in life expectancy with cardiovascular disease attributable to the Polymeal was 2.4 years. The reductions were similar for women, although the magnitudes were lower (table 2).

\section{Adverse effects}

No proved serious adverse effects were reported in any of the papers selected. For garlic, in addition to body odour, some unproved adverse effects were mentioned: flatulence, oesophageal and abdominal pain, allergic reactions, and bleeding. ${ }^{11}$ Fish consumed in larger amounts than recommended as part of the Polymeal has been related to raised blood mercury concentrations, especially with large fish such as shark and swordfish. ${ }^{7}$ No association between wine consumption at the level included in the Polymeal and increased risk of breast cancer was reported by the authors of the papers included in our analyses. ${ }^{6}$ 


\section{Discussion}

The Polymeal is an effective, natural, probably safer, and tastier alternative to the Polypill to reduce cardiovascular disease and increase life expectancy in the general population. The effect was consistent in both men and women at age 50 . Adverse effects reported for garlic include malodorous breath and body odour. ${ }^{13}$ As garlic is destined for mass treatment, few people will still notice this after a while. No additional adverse effects should be expected from the other ingredients of the Polymeal (in the quantities recommended here) except in people who are allergic to the components. Another advantage of the Polymeal is that its ingredients can be taken combined as a meal or individually at different times of the day. Taking the Polymeal on a daily basis (fish two to four times a week) should be feasible, considering that the ingredients are generally well tolerated and appreciated among the general population. The development and distribution of specific recipes combining the Polymeal ingredients could enhance the compliance of the population.

\section{Costs and precautions}

Although the exact price of the Polymeal is unknown and will be country specific, it could be expected to be similar to or perhaps higher than that of the Polypill. By checking a local supermarket in Rotterdam, the Netherlands, we estimated a total price for the Polymeal of $€ 21.60$ ( $£ 15.20 ; \$ 28.10)$ a week ( $€ 3.50$ for the wine, $€ 6.23$ for fruit and vegetables, $€ 2.80$ for almonds, €4.34 for dark chocolate, €0.14 for garlic, and $€ 4.60$ for fish). Although we do not recommend particular brands, spending more-for example, on your favourite bottle of wine or brand of chocolatemight also be rewarded by an improved quality of life.

The Polymeal should not be combined with additional consumption of alcohol, in order to avoid intoxication and conflicts with friends, relatives, and authorities; furthermore, additional alcohol consumption could attenuate the effects of the Polymeal and negatively influence other health measures. Driving motor vehicles or performing activities that require high levels of attention shortly after the consumption of the Polymeal should be avoided. Moreover, considering the disturbing adverse effects of garlic, we do not recommend taking the Polymeal before a romantic rendezvous, unless the partner also complies with the Polymeal.

We believe our search was comprehensive and although we looked for additional ingredients to include in the Polymeal, we found no other potential components with a sufficient level of evidence or with clearly reported effects on cardiovascular disease events or on modification of risk factors of cardiovascular disease. Some other ingredients could be added to the Polymeal (olive oil, echium oil, soya oil, soya beans, tomatoes, oat bran, cereals, nuts, tea, chickpeas, and so on), but this will only improve its effect on cardiovascular disease risk reduction.

Concerns might be raised about the validity of the source evidence and the multiplicative model used to calculate effects of the ingredients of the Polymeal. However, these are shared by the Polypill analyses, as we used a similar approach. None the less, a greater possibility of interaction exists between dietary factors

\section{What is already known on this topic}

Prevention of cardiovascular disease is limited by high costs and low compliance

The concept of a combination pill (the Polypill) to reduce cardiovascular disease by more than $80 \%$ was introduced in 2003

Pharmacological interventions are not the only option for preventing heart disease; a healthy diet and an active lifestyle reduce cardiovascular disease as well

\section{What this study adds}

A combined meal of seven food components (the Polymeal) could reduce cardiovascular disease by more than $75 \%$

Chocolate, wine, fish, nuts, garlic, fruit, and vegetables are all known to have a positive effect on cardiovascular disease, and have been enjoyed by humankind for centuries

Finding happiness in a frugal, active lifestyle can spare us a future of pills and hypochondria

as less information is available about underlying mechanisms of action. This might result in an overestimation of the effect of the Polymeal.

Another potential limitation of our study is that no back flows are allowed in the multi-state life tables, and only the first entry into a state is considered. This is not always seen in real patterns of morbidity and mortality.

No contraindications to combining the Polymeal with additional interventions seem to exist. After the daily consumption of the Polymeal, for example, half an hour of walking could prevent further cardiovascular disease events. ${ }^{21}$ For those people earnestly seeking to prevent cardiovascular disease, the Polypill can be combined with the Polymeal. The fortification of flour with Polypill ingredients (a statin, two antihypertensive drugs instead of three, folic acid, and aspirin) certainly merits further study. Redundant cardiologists could be retrained as Polymeal chefs and wine advisers.

\section{Conclusions}

The preventive strategy outlined here is radical. But the "healthy person" is an outdated concept from the era before scientific prevention. We should recognise that in Western society we all have cardiovascular risk factors, so everyone is at risk, and the diseases they cause are common and often fatal. It may be argued that the Polypill is even more effective, but the Polymeal promises to be an effective, nonpharmacological, safe, and tasty alternative for reducing cardiovascular morbidity and increasing life expectancy in the general population.

We thank the Framingham Heart Study Coordinators for access to the original dataset. The Framingham study is conducted and supported by the National Heart, Lung, and Blood Institute (NHLBI) in collaboration with the Framingham Heart Study Investigators. This manuscript has been reviewed by the NHLBI for scientific content and consistency of data interpretation with previous Framingham publications. We also thank M E Kruijshaar and L J Veerman for their valuable comments and A 
A Mamum and F Willekens for their collaboration in the understanding of the life table approach.

Contributors: All authors participated actively in conception and design of the study or analysis and interpretation of data, in drafting the article or revising it critically for important intellectual content, and in final approval of the version to be published. $\mathrm{OHF}$ is the guarantor.

Funding: This study was supported by grants from the Netherlands Heart Foundation (grant no 98.138) and the Netherlands Organization for Scientific Research (grant no 904-66-093). OHF, LB, CdL, AP, EWS, and JPM were partly funded by the Netherlands Heart Foundation (grant no 98.138) and the Netherlands Organization for Scientific Research (grant no 904-66-093). AP was also partly funded by VicHealth (fellowship grant no 2002-0191). All authors have acted independently from the funders of this project.

Competing interests: None declared.

Ethical approval: Not needed as this was a secondary data analysis.

1 Gluckman TJ, Baranowski B, Ashen MD, Henrikson CA, McAllister M, Braunstein JB, et al. A practical and evidence-based approach to cardioBraunstein JB, et al. A practical and evidence-based approach to car

2 Wald NJ, Law MR. A strategy to reduce cardiovascular disease by more than $80 \%$. BMJ 2003;326:1419-23.

3 Correspondence. "Polypill" to fight cardiovascular disease. BMJ 2003;327:807-10.

4 Sacket DL, Straus SE, Richardson WS, Rosenberg W, Haynes RB Evidence-based medicine: how to practice and teach EBM. New York: Churchill Livingstone, 2000.

5 Oxford Centre for Evidence Based Medicine. Levels of evidence and grades of recommendation. www.cebm.net/levels_of_evidence.asp (accessed 5 Aug 2004)

6 Di Castelnuovo A, Rotondo S, Iacoviello L, Donati MB, De Gaetano G. Meta-analysis of wine and beer consumption in relation to vascular risk. Circulation 2002;105:2836-44

7 Whelton SP, He J, Whelton PK, Muntner P. Meta-analysis of observational studies on fish intake and coronary heart disease. Am I Cardiol 2004;93:1119-23.
Taubert D, Berkels R, Roesen R, Klaus W. Chocolate and blood pressure in elderly individuals with isolated systolic hypertension. JAMA 2003;290:1029-30.

9 Neal B, MacMahon S, Chapman N. Effects of ACE inhibitors, calcium antagonists, and other blood-pressure-lowering drugs: results of prospectively designed overviews of randomised trials. Lancet 2000;356:1955-64

10 John JH, Ziebland S, Yudkin P, Roe LS, Neil HA. Effects of fruit and vegetable consumption on plasma antioxidant concentrations and blood pressure: a randomised controlled trial. Lancet 2002;359:1969-74.

11 Ackermann RT, Mulrow CD, Ramirez G, Gardner CD, Morbidoni L, Lawrence VA. Garlic shows promise for improving some cardiovascular risk factors. Arch Intern Med 2001;161:813-24.

12 Silagy C, Neil A. Garlic as a lipid lowering agent-a meta-analysis. J $R$ Coll Physicians Lond 1994;28:39-45.

13 Law MR, Wald NJ, Thompson SG. By how much and how quickly does reduction in serum cholesterol concentration lower risk of ischaemic heart disease? BMJ 1994;308:367-72

14 Berthold HK, Sudhop T, von Bergmann K. Effect of a garlic oil preparation on serum lipoproteins and cholesterol metabolism: a randomized controlled trial. JAMA 1998;279:1900-2.

15 Jenkins DJ, Kendall CW, Marchie A, Parker TL, Connelly PW, Qian W, et al. Dose response of almonds on coronary heart disease risk factors: blood lipids, oxidized low-density lipoproteins, lipoprotein(a) homocysteine, and pulmonary nitric oxide: a randomized, controlled, crossover cysteine, and pulmonary nitric oxide:

16 Sabate J, Haddad E, Tanzman JS, Jambazian P, Rajaram S. Serum lipid response to the graduated enrichment of a step I diet with almonds: a
randomized feeding trial. Am J Clin Nutr 2003;77:1379-84.

17 Dawber TR, Meadors GF, Moore FE Jr. Epidemiological approaches to heart disease: the Framingham study. Am J Public Health 1951;41:279-81.

18 Stokes J 3rd, Kannel WB, Wolf PA, Cupples LA, D'Agostino RB. The relative importance of selected risk factors for various manifestations of cardiovascular disease among men and women from 35 to 64 years old: 30 years of follow-up in the Framingham study. Circulation 1987;75:V65-73.

19 De Backer G, Ambrosioni E, Borch-Johnsen K, Brotons C, Cifkova R, Dallongeville J, et al. European guidelines on cardiovascular disease prevention in clinical practice. Eur Heart J 2003:24:1601-10.

20 Peers A, MamunAA, Willekens, Bonne L A cadiorsculo life history: a life course analysis of the original Framingham heart study cohort. Eur Heart J 2002;23:458-66.

21 US Department of Health and Human Services. Physical activity and health: a report of the surgeon general. Atlanta: Centers for Disease Control and Prevention, National Center for Chronic Disease Prevention and Health Promotion, 1996

(Accepted 4 November 2004)

\section{Randomised controlled trial of magnetic bracelets for relieving pain in osteoarthritis of the hip and knee}

College Surgery, Cullompton, Devon

EX15 1TG

Tim Harlow

general practitioner

Peninsula Medical

School (Primary

Care), Exeter

EX2 5DW

Colin Greaves

research fellow

Peninsula Medical

School, Tamar

Science Park,

Plymouth PL6 8BX

Adrian White

senior research fellow

Peninsula Medical

School

(Complementary

Medicine), Exeter

EX2 4NT

Liz Brown

research assistant

Edzard Ernst

professor of

complementary

medicine

continued over

BMJ 2004;329:1450-4

Tim Harlow, Colin Greaves, Adrian White, Liz Brown, Anna Hart, Edzard Ernst

\section{Abstract}

Objective To determine the effectiveness of commercially available magnetic bracelets for pain control in osteoarthritis of the hip and knee.

Design Randomised, placebo controlled trial with three parallel groups.

Setting Five rural general practices.

Participants 194 men and women aged 45-80 years with osteoarthritis of the hip or knee.

Intervention Wearing a standard strength static bipolar magnetic bracelet, a weak magnetic bracelet, or a non-magnetic (dummy) bracelet for 12 weeks. Main outcome measures Change in the Western Ontario and McMaster Universities osteoarthritis lower limb pain scale (WOMAC A) after 12 weeks, with the primary comparison between the standard and dummy groups. Secondary outcomes included changes in WOMAC B and C scales and a visual analogue scale for pain.

Results Mean pain scores were reduced more in the standard magnet group than in the dummy group (mean difference 1.3 points, $95 \%$ confidence interval
0.05 to 2.55 ). Self reported blinding status did not affect the results. The scores for secondary outcome measures were consistent with the WOMAC A scores. Conclusion Pain from osteoarthritis of the hip and knee decreases when wearing magnetic bracelets. It is uncertain whether this response is due to specific or non-specific (placebo) effects.

Manufacturers of permanent static magnet devices claim that they reduce pain in various conditions, including osteoarthritis. ${ }^{1}$ Worldwide sales were estimated at \$5bn (£2.6bn, €3.8bn) in 1999. ${ }^{2}$ Osteoarthritis affects around 760000 people in the United Kingdom, producing an estimated 3.02 million general practice consultations in 2000. If magnets were effective they would offer a cheap and probably safe treatment option.

Some studies of permanent static magnets have found significant pain reduction ${ }^{2-9}$ whereas others reported no effect. ${ }^{10-12}$ Major differences exist in the type and strength of magnets used, the conditions

A chart showing flow of participants is on bmj.com 